\title{
Historia de una idea invisible: La no presencia de los conceptos de construcción de paz y reconciliación en la teoría sobre la violencia en Perú
}

\section{History of an Invisible Idea: The Non- Presence of the Concepts of Peace Building and Reconciliation in the Theory of Violence in Peru}

\author{
Egoitz Gago Antón ${ }^{1}$ \\ Pontificia Universidad Javeriana (Colombia)
}

Recibido: 01-08-19

Aprobado: 06-03-20

\section{Resumen}

Perú es un caso claro de conflicto interno violento. Este conflicto, dominado por las acciones del grupo guerrillero Sendero Luminoso tuvo sus años más duros durante las décadas de los años 80 y 90 . Sin embrago, es peculiar la poca producción académica que existe analizando los aspectos de paz y conflicto del país. Este trabajo busca repasar los trabajos realizados en el país sobre la violencia y analizar la existencia de contribuciones académicas que traten el conflicto peruano desde la perspectiva de los estudios de paz y conflicto. Para ello se presentan los conceptos de construcción de paz y reconciliación según los autores seminales de los estudios de paz y se contrastan esos conceptos con las contribuciones académicas más destacadas. Este artículo concluye que existe un cuerpo limitado de trabajo al respecto, generado en dos épocas diferenciadas.

\footnotetext{
${ }^{1}$ (egoitz-gago@javeriana.edu.co) Doctor en Estudios de Paz por la Universidad de Bradford. Profesor Asociado de planta del Departamento de Ciencia Política de la Pontificia Universidad Javeriana de Bogotá, Colombia. Sus últimas publicaciones son: La contribución social a la paz en Euskadi. Madrid: La Catarata, 2017 y. "¿Cómo se articula la acción social en entornos de conflicto? El caso de San Basilio de Palenque". Araucaria. Revista Iberoamericana de Filosofia, Política y Humanidades, 20(39), 2018, pp. 467-489.

ORCID: https://orcid.org/0000-0003-1064-6377.
} 
Durante los años de la violencia serán trabajos que cuestionan las acciones de Sendero Luminoso y después de la creación de la Comisión de la Verdad aparecerán trabajos más alineados con los estudios de paz, sobre todo con los conceptos de memoria y reconciliación.

Palabras-clave: Perú, Construcción de Paz, Reconciliación, Teoría del conflicto, Sendero Luminoso.

\begin{abstract}
Peru is a clear case of internal violent conflict. This conflict has its peak during the eighties and the nineties, dominated by the actions of the Shining Path guerrilla movement. However, it is interesting to note the few academic works generated analyse the peace and conflict dimensions. This academic article seeks to review the different works produced in Peru regarding violence and to analyse the different contributions from the Peruvian academia regarding peace and conflict. To that task, there will be a review of the theoretical concepts of peacebuilding and reconciliation presented by the seminal authors in the peace studies field and compared with the most relevant contributions from the Peruvian academia regarding violent conflict. This article concludes that there is a very limited body of knowledge regarding violence. Moreover, this body of knowledge belongs in two different timeframes. During the hardest violent years, most of the academic works focused on the Shining Path's actions and its legitimacy. After the creation of the Truth and Reconciliation Commission, a new body of knowledge appeared, closer to the concepts of reconciliation, especially dealing with memory and truth.
\end{abstract}

Key-words: Peru, Peacebuilding, Reconciliation, Conflict Theory, Shining Path.

\title{
1. Introducción
}

Los entornos de conflicto prolongado son un caldo de cultivo de donde surgen estudios intelectuales sobre la naturaleza de dicho conflicto, las causas de la presencia de la violencia y posibles propuestas para su resolución. El caso peruano presenta unas contradicciones al respecto que son muy interesantes de analizar. A diferencia de otros estudios de caso en la región, durante los años más duros de la violencia en el Perú (1980-1999), no se observa una producción prolífica en los temas de paz y conflicto. Los trabajos que surgen de las ciencias sociales al respecto buscan analizar los efectos de la violencia desde una perspectiva crítica hacia la existencia de el grupo armado insurgente más importante del país, Sendero Luminoso. 
Este trabajo busca observar cómo la producción académica peruana deja de lado los trabajos sobre conflicto y paz y se centra en analizar la estabilidad de las instituciones peruanas y la amenaza que conlleva la existencia de Sendero Luminoso.

Para ello se planteará un ejercicio dividido en dos partes diferenciadas. Por un lado, se presentarán los conceptos básicos de construcción de paz y reconciliación tal y como aparecen en la teoría de los estudios de paz. Para ello se utilizarán los trabajos de autores seminales como Johan Galtung y John Paul Lederach, así como a portaciones más novedosas que han surgido al respecto. En la presentación de esos conceptos se constatará como los estudios de paz y conflicto necesitan, de manera imperiosa, el reconocimiento de las partes involucradas en la violencia para poder proponer caminos de entendimiento que eviten el uso de la mencionada violencia. Por otro, se hará un repaso a las aportaciones más destacadas sobre el conflicto armado interno en el Perú, diferenciando entre el periodo duro de la violencia (1980-1999) y el periodo que surge tras el descabezamiento de Sendero Luminoso y la creación de la Comisión de la Verdad y la Reconciliación en 2001.

La comparación de estos dos apartados nos dará una imagen del campo de los estudios de paz referentes al conflicto peruano. En efecto, se observará que existe un déficit en la producción académica al respecto, con un repunte importante después de 2001. La aparición y generación de informes por parte de la Comisión de la Verdad y la Reconciliación y las acciones ocurridas durante el gobierno de Alberto Fujimori (1990-2000) impulsarán un núcleo de contribuciones académicas centradas en el análisis de la memoria y el relato de la violencia, así como el análisis crítico de las acciones del Estado durante los años del conflicto. Además, se podrá observar la aparición de trabajos que incluirán los conceptos de construcción de paz y reconciliación defendidos por los estudios de paz. Este artículo concluirá que, si bien ha habido un repunte en la producción académica de paz y conflicto en el Perú, este escenario tiene todos los ingredientes para generar un cuerpo teórico propio referente a los estudios de paz y el análisis y resolución de conflictos.

Finalmente, es importante recalcar que este trabajo genera más preguntas y respuestas. Si bien se presenta el hecho de la existencia de un déficit en la producción de estudios de paz en el Perú, no entra de lleno en responder los motivos por los cuales eso ocurre. Para ello se presentan sugerencias de varios autores peruanos, en especial Carlos Iván Degregori, que apunta a la fragmentación y carácter poscolonial de la academia peruana. Se anima, por lo tanto, a profundizar los motivos de este vacío, así como a la producción académica profunda en el Perú sobre los estudios de paz y conflicto. 


\section{Hacia una aproximación de los conceptos de construcción de paz y reconciliación}

La literatura académica es prolífica a la hora de explicar a qué nos referimos cuando hablamos de construcción de paz. Sin embargo, este concepto teórico, ha sido usado de manera indiscriminada. En este trabajo se presenta de forma crítica las distintas teorías que tratan el concepto de construcción de paz, así como sus conceptos adjuntos, como son posconflicto y reconciliación. Para ello se consideran los trabajos teóricos, dentro de la disciplina de estudios de paz realizados por Johan Galtung (1998, 2003), John Paul Lederach (1998, 2005), Oliver Ramsbothan, Tom Woodhouse y Hugh Miall (2015; 2011), Vincenc Fisas (2002), Chester Crocker, Fen Osler Hampson y Pamela All (2001, 2015) y Alpaslam Özerdem (2016). Estas aportaciones teóricas, que presentan los cimientos de los conceptos de construcción de paz, serán complementadas por trabajos con dimensión empírica, ya que la construcción de paz y sus conceptos adjuntos son elementos dinámicos de las sociedades divididas. Las aportaciones de autores como Oliver Richmond (2005), con su concepto de paz posliberal, Roger Mac Ginty o Alpaslam Özerdem (2019), ofrecen una apreciación de la aplicación práctica de los conceptos de construcción de paz, reconciliación y posconflicto.

Los estudios de construcción de paz entienden el conflicto como algo inherente a la sociedad. Para avanzar en nuestro análisis, es importante hablar de tres conceptos: qué es el conflicto, qué es la resolución del conflicto y qué es la violencia. El conflicto es una fuerza intrínseca de nuestro desarrollo social y no debe ser rechazado. Este enfoque, que viene de los estudios de conflicto social defendidos a mitad del siglo pasado por Ralf Dahrendorf (1968) y Lewis Coser (1956), entiende el conflicto como algo positivo, que ayuda a que las sociedades modernas avancen, rechazando los enfoques estáticos funcionalistas de la época defendidos, sobre todo, por Talcott Parsons y Robert K. Merton.

El enfoque central del conflicto social es el adoptado por los teóricos de los estudios de paz en los años 80 y 90, enfocándose no en la existencia o no del conflicto, sino en su manejo. El problema, por lo tanto, va a centrarse en que hace del conflicto una herramienta destructiva (Vinyamata, 2001; Fisas, 2002). La resolución de conflictos (Galtung, 1998) o la transformación de conflictos (Lederach, 1998), se va a centrar en los distintos procesos y herramientas que se deben abordar para que el conflicto sea un elemento constructivo. La gran diferencia será en la existencia o no de violencia. Un conflicto será constructivo cuando no exista violencia o los elementos violentos se traten de manera comprensiva. Si no es así, estaremos tratando con un conflicto destructivo.

Los procesos de construcción de paz estarán protagonizados por las dinámicas que tratarán los distintos aspectos de la violencia. Para Johan 
Galtung (1998) la violencia es todo aquello que impide el desarrollo normal del ser humano. El autor divide la violencia en tres dimensiones: la violencia directa que es aquella que se suele asociar como violencia al ser visible y buscar el daño físico, la violencia estructural, que surge de las estructuras sociales y políticas, y la violencia cultural, que legitima la violencia mediante los valores. En un conflicto destructivo, todas estás dimensiones se entremezclan entre sí. La violencia no es homogénea, es dinámica y se presenta con distintas caras según la forma de conflicto que nosotros podamos observar. Por lo tanto, la idea que existe y que va a protagonizar todos los estudios del conflicto van a abandonar la categoría de conflicto como unidad de análisis, ya que el problema no va a ser la existencia o no del mismo, sino la existencia o no de los distintos aspectos de la violencia. Esto presenta una primera afirmación importante: para entender la construcción de paz, se debe evitar el término posconflicto, pues este nunca deja de existir (Ríos y Gago, 2015, 2018). El enfoque académico, por lo tanto, se hará en los elementos que ayudan a que los conflictos no sean violentos, sino pacíficos.

\section{La construcción de paz en la teoría académica}

Como se ha adelantado anteriormente, se ha entendido el térmico de construcción de paz como algo que ocurre "después" del conflicto. Nada más lejos de la realidad, la construcción de paz o peacebuilding incluye procesos de gran complejidad que ocurren durante y después de la existencia de la violencia. Estos procesos incluyen dinámicas como la reforma del estado, construcción de nación política, o sanación de las sociedades divididas. Una característica común de los autores que tratan este concepto es que la construcción de paz culmina con un cambio sustancial de la cultura que sostiene la violencia, creando lo que algunos autores denominan cultura de paz (Vinyamata, 2001; Fisas, 2002; Gago, 2016).

Si bien los autores comparten elementos comunes, existe una gran divergencia de opiniones sobre cuales son las fases de la construcción de paz. Por ejemplo, el propio término de reconstrucción no está exento de problemas. Mary Fitzduff, en su trabajo sobre el papel de la sociedad civil en la construcción de paz en Irlanda del Norte, invita a dar una mirada crítica al ese concepto, intentando escapar de sus acepciones mas clásicas que invitan volver al pasado (Fitzduff, 2002). Otros autores, en cambio, defienden la existencia de una reconstrucción que de derecho a las víctimas a recuperar sus vidas perdidas por la violencia (Ashplant et al., 2004). Lo único que une a todos los autores es el carácter de cambio que tiene la construcción de paz. 
No se trata de volver al pasado ni de recrear las condiciones que se daban antes de la aparición de la violencia. La construcción de paz implica una revisión profunda de las causas de esa misma violencia para que no se vuelvan a dar y construir una nueva comunidad política en la que la necesidad del uso de esa violencia no tenga espacio en el imaginario colectivo. El conflicto persiste y estará presente, pero será resuelto por procesos inclusivos y pacíficos. Como se ha explicado, el objetivo final del proceso de construcción de paz es conseguir una paz sostenible y duradera. Uno de los enfoques más desarrollados sobre la capacidad operativa de los procesos de construcción de paz es el defendido por Tom Woodhouse, Oliver Ramsbothan y Hugh Miall (2011). Los autores defienden el proceso de construcción de paz dividido en cuatro aspectos diferenciados:

1. Seguridad. Engloba todos los procesos relacionados con el desarme, la desmovilización y reintegración (DDR) de agentes armados, así como la reforma del sector seguridad, el desminado humanitario y la regulación sobre la existencia de armas ligeras.

2. Marco político. Incluye todo aquello referente a las reformas políticas que deben ocurrir en un entorno de construcción de paz como democratización de partidos políticos, creación de cultura democrática, refuerzo del estado de derecho, fortalecimiento de las instituciones políticas y todo lo que tenga que ver con los derechos humanos.

3. Fundamentos socioeconómicos. Este aspecto incluye todo lo referente a la construcción de la seguridad económica como infraestructuras, repatriación y retorno de desplazados y seguridad alimentaria.

4. Reconciliación y justicia. Todo lo que tenga que ver con entendimiento y dialogo entre las partes enfrentadas, creación de procesos de justicia transicional y comisiones de la verdad, tratamiento del trauma y atención a víctimas. (Woodhouse et al., 2011: 229).

Estos cuatro puntos son los que aparecen en todo acuerdo de paz firmado en el último medio siglo, con mayor o menor importancia (Fisas, 2010). Sin embargo, estos aspectos también deben ser aplicados de forma cautelosa. Conviene recordar que es necesario la inclusión del Estado y de las instituciones políticas para una correcta aplicación de estos puntos, pero sin caer en una mera lista. Hacerlo provocaría un proceso de construcción de paz incompleto o fallido, como apuntan autores como Roger Mac Ginty (Mac Ginty, 2006), Oliver Richmond (Richmond, 2005) o Alpaslam Özerdem (Özerdem y Mac Ginty, 2019). Este último autor presenta una relación de procesos de paz fallidos debido a este problema. Por lo tanto, la construcción de paz no solamente necesita un acercamiento a los procesos explicados, también implica una reimaginación del propio Estado a todos los niveles. La construcción de paz debe venir con una renovación de las relaciones políticas, sociales y afectivas, necesarias para poder comprender a la otra parte de igual a igual (Lederach, 2005; Lederach y Lederach, 2014). 
El trabajo más amplio realizado sobre la construcción de paz ha sido elaborado por el teórico estadounidense John Paul Lederach. Este autor presenta una ruta clara sobre los distintos procesos que se deben llevar a cabo en un entorno de construcción de paz. Su obra más determinante al respecto es Construyendo la paz. Reconciliación sostenible en sociedades divididas (1998). En esta obra, eparte de la enorme complejidad que existe en las sociedades divididas por la violencia, por lo que la construcción de paz debe de superar las aproximaciones clásicas y tratar estos conflictos de forma muy parecida a los elementos que defienden autores como Mary Kaldor y su teoría de las "nuevas guerras" (Kaldor, 1998).

Los conflictos descritos por Lederach están caracterizados por un gran antagonismo que impide la existencia de herramientas de acercamiento para abordar dicho antagonismo (Lederach, 1998). Además, este antagonismo está relacionado por una distribución de poder entre varios actores que lo perciben como insuficiente. Para tratar estos escenarios, el autor sugiere la búsqueda de mecanismos innovadores que den respuesta a los problemas planteados. Para ello se planeta la limitación del poder del Estado (normalmente un actor más de la violencia) y la reparación de las relaciones de poder y sociales. Esto desemboca en un proceso de construcción de paz integral que repercute en todos los aspectos de una comunidad política. Así, para Lederach, la construcción de paz es de largo aliento y requiere de cuatro fases diferenciadas:

1. Intervención en la crisis: Esto es, acción inmediata sobre los efectos de la violencia.

2. Preparación y formación: Planificación a corto plazo para paliar las consecuencias inmediatas de la violencia.

3. Diseño del cambio social: Proceso de cambio a una década que busca reformar las relaciones sociales.

4. Sistema/relación. Objetivos y definición: Consecución de la cultura de paz en las próximas generaciones.

Además, para evitar los problemas apuntados anteriormente, Lederach presenta un modelo de trabajo con tres niveles de actores relacionados entre sí: 


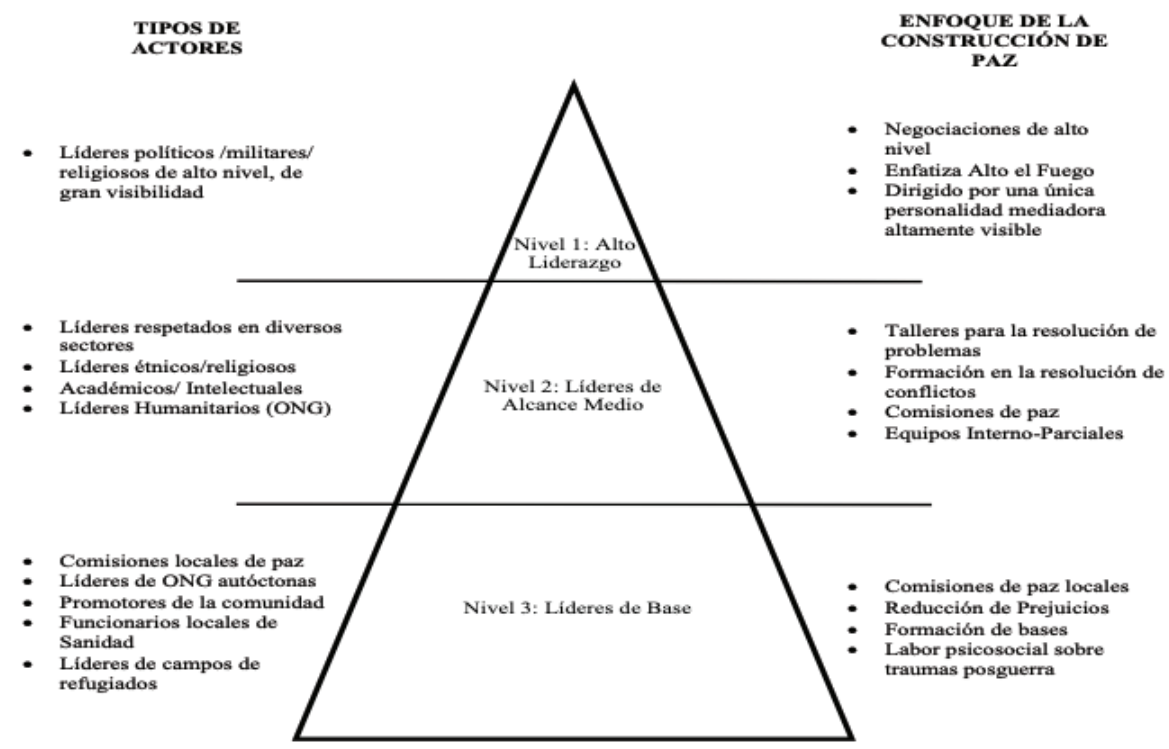

Fuente: Lederach, 1998: 66.

Todas las fases del proceso de construcción de paz deben realizarse de forma integrada entre todos los actores presentados por el autor. Estos actores dependen de su posición de liderazgo y cada uno tendrá funciones diferentes: los actores de alto nivel llevaran a cabo procesos de negociación y diplomacia, los de nivel medio trabajaran procesos de mediación y educación para la paz, mientras que los líderes de base tendrán como función más importante la acción psicosocial sobre comunidades afectadas por la violencia. Lederach defiende un proceso de construcción de paz inclusivo. Sin la presencia de todos los actores de manera integrada, dicho proceso de paz estaría propenso al fracaso.

En resumen, el proceso de construcción de paz para John Paul Lederach trata de abordar problemas y escenarios de suma complejidad que hacen necesaria un modelo de acercamiento individualizado. Esto implica entender, de manera exhaustiva, los distintos elementos que causan la violencia, así como sus consecuencias, para poder ser tratadas de forma adecuada. Lo anterior, en tanto que la construcción de paz es un proceso holístico, en donde todos los actores deben estar involucrados. Para ello, el autor identifica tres tipos de actores dependiendo de su cercanía con el proceso de toma de decisiones políticas y su posición de liderazgo: alto, medio y de base. Estos actores tienen características diferentes, pero de igual importancia para enfrentar el proceso 
de construcción de paz. El objetivo final del autor es alcanzar la reconciliación, un momento en el que las heridas sociales se hayan sanado y se alcance una cultura de paz plena.

\section{La reconciliación: El objetivo final de la construcción de paz}

La producción académica sobre la construcción de paz apunta a un escenario deseado: la reconciliación. Este término es muy usado en los distintos entornos de conflicto, pero su trato teórico es muy particular. Johan Galtung define la reconciliación como el proceso que debe darse para solventar los procesos de violencia cultural (1998). Si bien el autor resalta la importancia de realizar procesos que traten los efectos de los tres tipos de violencia (directa, estructural y cultural), el enfoque de reconciliación se expone como cierre. La reconciliación, por lo tanto, se presenta como un proceso de curación de las estructuras sociales dañadas por el conflicto. Es todo proceso a partir del cual las personas se sienten actores claves en el proceso de construcción de paz y ven sus valores transformados. Para ello, Galtung sugiere siete maneras distintas de enfocar la reconciliación:

1. Enfoque exculpatorio o inculpatorio, en el que la responsabilidad del daño causado se abandona totalmente o se asume de forma integral por todos los actores involucrados.

2. Enfoque de reparación/restitución, en el que el victimario repara el daño causado a la víctima.

3. Enfoque de disculpa/perdón, en el que existe una reparación cultural hacia la víctima.

4. Enfoque penitencial y punitivo, en el que el victimario se enfrenta a los modelos de justicia ordinaria basada en el castigo.

5. Enfoque histórico/teatral, en el que la memoria y el relato son parte central del proceso de reconciliación.

6. Enfoque conjunto, en el que todos los actores son parte del esfuerzo de construcción de paz.

7. Enfoque holístico u "Ho'oponopono", en el que tanto la víctima como el victimario se rehabilitan a través de una construcción conjunta del relato de los hechos. (Galtung, 1998: cap. VIII)

Estos enfoques no se presentan como excluyentes. El autor sugiere que cada proceso es particular y con características propias, por lo que deberán aplicarse los enfoques más adecuados para cada uno. Sin embargo, Galtung no es capaz de arrojar concreción al proceso de reconciliación. Un enfoque más aterrizado es presentado por Oliver Ramsbothan, Tom Woodhouse y Hugh Miall, que definen la reconciliación como la restauración de relaciones rotas 
y el aprender a vivir de forma no violenta asumiendo diferencias radicales (Woodhouse et al., 2011: 246). Para ello se enfocan en cuatro dimensiones importantes: el fin de la violencia, el superar la polarización, el manejar las contradicciones y, por último, celebrar las diferencias. Así, presentan los distintos aspectos que están presentes en los procesos de construcción de paz y reconciliación, como tratar el trauma de las víctimas y una somera descripción a los procesos de justicia transicional, donde el objetivo no es el imponer castigo, sino reconocer el daño causado. Finalmente, los autores explican que la reconciliación se alcanza utilizando métodos que reconocen el daño y celebran el relato, evitando procesos de olvido (amnesia oficial) y venganza (Woodhouse et al., 2011: 252-254).

Una de las conceptualizaciones de la reconciliación más completas la presenta el autor sueco Kjell-Ake Nordquist, que sugiere que la reconciliación debe ser vista como un proceso de cierre o una fase dentro del continuo del conflicto, sino una política integral que debe dominar todos los aspectos de un proceso de paz (Nordquist, 2018). Desde este punto de vista. La reconciliación debe de conceptualizarse desde el propios análisis y propuesta de resolución del conflicto. El relajamiento de las tensiones entre partes y actores de un conflicto violento no solamente se da desde un lugar concreto en la resolución, sino que debe guiar los procesos de construcción de paz. El objetivo, si bien no es algo infalible, es ser capaz de tratar todas las tensiones que existen y que pueden aparecer dentro de un proceso de paz. Incluir la reconciliación como política central dentro de un Estado ayuda a que la sanación tenga lugar con mayor probabilidad.

Finalmente, es importante resaltar las aportaciones realizadas por Chester Crocker, Fen Osler Hampson y Pamela All (2001, 2015). El enfoque de estos autores es mucho más instrumental que los presentados hasta ahora, al exponer en dos grandes obras editadas, los distintos pasos que han de darse ante un proceso de construcción de paz y reconciliación. Ante la pregunta de cómo se puede construir paz en un escenario de guerra prolongada, se presentan varios pasos concretos:

1. Asegurar la seguridad que permita la actividad económica.

2. Fortalecer la capacidad de acción del Estado.

3. Asistir el regreso de personas desplazadas.

4. Fortalecer las economías locales.

5. Recuperar el tejido comunitario.

6. Reconstrucción de las estructuras económicas.

7. Desminado humanitario.

8. Rehabilitar las instituciones financieras.

9. Reconciliación integral.

10. Priorizar zonas y grupos afectados por la violencia. (Ball, 2001: 723) 
El planteamiento de la autora se centra en la recuperación económica de las sociedades azotadas por la guerra. Si bien esta idea choca con las aproximaciones presentadas por Johan Galtung y John Paul Lederach, no hay que subestimar la necesidad de un sector seguridad saneado y una economía estable en entornos de construcción de paz. Varios de sus pasos son útiles para entender los procesos de reconciliación, sobre todo la reintegración de excombatientes y la participación de las instituciones multilaterales.

Por último, es importante hablar de los procesos de justicia que aparecen durante la construcción de paz. Para ello, uno de los enfoques más utilizados hoy en día es el de justicia transicional (Stromseth, 2015). Este enfoque busca generar espacios de verdad y reconocimiento del daño causado, básicos para llegar a los procesos de reconciliación. Para ello, presenta una tipología de distintos mecanismos como las comisiones de la verdad, los juicios criminales o las reparaciones (Stromseth, 2015: 574). Estos mecanismos, como la mayoría de los enfoques presentados, han sido utilizados de forma variable, como por ejemplo los procesos de cortes de justicia hibridas y las experiencias de justicia transicional a nivel local. Sin embargo, la justicia transicional, como muchos de los procesos presentados, no es la panacea. Este modelo se enfrenta a la tensión entre justicia y paz (Woodhouse et al., 2011) o las tensiones derivadas de la reforma del sector justicia en sociedades en proceso de construcción de paz (Stromseth, 2015).

\section{La presencia de los conceptos de construcción de paz y reconciliación en la literatura académica sobre la violencia en Perú}

En su trabajo Qué difícil es ser Dios, el Partido Comunista del PerúSendero Luminoso y el conflicto armado interno del Perú: 1980-1999, Carlos Iván Degregori (2011) apunta la sorpresiva ausencia de estudios intelectuales sobre el conflicto peruano hasta la conformación de la Comisión de la Verdad en 2001. El autor presenta unas razones para ello, haciendo hincapié en la característica poscolonial del país, así como la naturaleza rural del conflicto. Sin embargo, las causas son más profundas y se sugiere que estén centradas en la naturaleza de la violencia realizada por Sendero Luminoso en los años más duros del conflicto, entre 1980 y 1999.

En efecto, una rápida revisión de los textos más prominentes sobre el conflicto peruano durante esas fechas indica una gran existencia de obras que se centran en analizar la violencia (Ansión, 1989; Arce Borja, 1989; Basombrio, 1994; Biondi \& Zapata, 1989; Mac Gregor et al., 1985; Urbano, 1991), o distintos aspectos de ella sin entrar a valorar posibles escenarios de resolución basados en los conceptos de reconciliación o construcción de paz presentados anteriormente. 
La mayoría de los trabajos presentados en la época más dura del conflicto peruano se centran en la situación jurídica de las acciones guerrilleras, haciendo énfasis en la suave respuesta del sistema jurídico peruano al respecto (Ambos, 1999) o en las acciones delictivas de Sendero Luminoso (Dreyfus, 1999).

Una de las explicaciones que pueden aparecer al respecto es la no existencia de procesos de paz en esos años en el Perú, a diferencia de lo ocurrido en otros escenarios de conflicto armado en América Latina, como El Salvador (González Marrero y Harto de Vera, 1994) o Colombia (GarciaDurán, 2004). Este hecho, unido a los aspectos presentados anteriormente en la obra de Degregori (1997, 2011), hacen difícil la aparición de contribuciones relacionadas con la construcción de paz y la reconciliación en la producción intelectual que trata el conflicto armado en el Perú.

Los únicos apuntes que podrían relacionarse con los conceptos presentados en el apartado anterior habría que buscarlos en contribuciones que no tocan directamente los aspectos violentos del conflicto, sino temas tangentes, como los procesos de democratización y el desarrollo de nuevos movimientos sociales a nivel global (Apter, 1993). Una excepción importante, ya que aparece durante la época más dura de la violencia, es la planteada por Susana Kaufman al centrar la necesidad de procesos de espeto a la memoria y tratamiento del trauma en los procesos violentos de América Latina, mencionando como caso a Perú (Kaufman, 1998). Si bien su aportación se hará patente durante las fechas posteriores a la derrota de Sendero Luminoso y la aparición de la Comisión de la Verdad, no deja de ser llamativa la aparición de la contribución de la autora con anterioridad a 1999.

Los intentos de proponer un cuerpo académico relacionado con la construcción de paz vendrán de los esfuerzos del Padre Felipe Mac Gregor, después de serle encargado el proceso de generar una asociación que avance en el estudio de la paz en el Perú. Sin embargo, sus esfuerzos irán en una dirección clara: la generación de una cultura de paz (Mac Gregor, 1986), pero sin incluir los procesos de reconciliación ni reconocimiento del otro. La violencia de Sendero Luminoso no será vista como algo que surge de unas causas estructurales, sino algo a evitar y, por lo tanto, no asumible dentro del cuerpo académico de la época.

Este enfoque se irá complementado con distintas contribuciones destinadas a la educación para la paz (Ansión, 1992; Bolaños, 1992; Díaz Bazo, 1996). El enfoque defendido por el padre Mac Gregor es interesante, al estar encargado de los estudios de paz en el Perú a través de la fundación en 1983 de la Asociación Peruana de Estudios de Paz. Esta asociación se fundó con el objetivo de "llevar a cabo actividades tendientes a profundizar el conocimiento y difundir la importancia de la ciencia de la paz" (Giesecke, 1993). Sin embargo, a diferencia de otros centros en la región, la asociación peruana no abordará la 
necesidad de una construcción de paz inclusiva, sino una necesidad de eliminar la violencia, en concreto la violencia insurgente. Este hecho llevará a que un gran cuerpo del trabajo académico de la época se centre en la legitimidad de las acciones del grupo insurgente, centrando sus fundamentos de acción, su relación con los colectivos que defiende y sus objetivos (Ansión, 1982; Berg, 1986; Burga, 1988; Palmer, 1992; Castañeda, 1993).

Los trabajos sobre la organización armada durante esa época se centrarán, como se ha comentado anteriormente, en su conexión con sus bases fundacionales, sus objetivos y su legitimidad. Normalmente se presentarán como algo ilegítimo (De Soto, 1986; Mason \& Campany, 1995; Degregori, 199) o se intentará abordar el tema desde un punto de vista neutral, describiendo de forma objetiva los diferentes hechos violentos (DESCO, 1989). Una vez más, esta característica es algo que diferencia a otros entornos de conflicto armado. Durante los años de la violencia en Colombia surgirán textos en los que, además de explicar la naturaleza de los distintos grupos armados, se propondrá su ideario ideológico de forma comprensiva y se asumirá una posición de inclusión sobre un hipotético proceso de construcción de paz (Medina Gallego, 2010; Valenzuela, 2014). La razón de esta descompensación en la producción intelectual de la época en Perú se intentará paliar con distintos estudios que incidirán en la naturaleza urbana del liderazgo de Sendero Luminoso, en contraste con su vocación rural (Poole y Rénique, 1991; Strong, 1992). Así, es interesante recalcar que los estudios académicos que buscan justificar la acción del grupo armado son escasos y se limitan a vincular su organización y accionar con su carácter de izquierda revolucionaria.

En resumen, la mayoría de producción intelectual generada durante los años de la violencia más dura en el Perú van a tener un déficit manifiesto en conceptos que surgen de los estudios de paz y conflicto. Los enfoques en cultura de paz y reconciliación presentados anteriormente no van a aparecer, incluso sugiriéndose un hecho premeditado para no aplicarlos al caso (Degregori, 2011). Los estudios sobre el conflicto se verán limitados a explicar los efectos de la violencia en las comunidades afectadas, sobre todo campesinos e indígenas. Además, surgirán multitud de estudios que pondrán en cuestionamiento la legitimidad de las acciones de Sendero Luminoso o, como mucho, harán una recapitulación de los hechos violentos. La única aportación hacia los estudios de paz de la época será la contribución del padre Felipe Mac Gregor, pero se verá limitada a una exposición de los conceptos de cultura de paz y educación para la paz.

Estas dinámicas cambiarán de forma importante después de la derrota de Sendero Luminoso. El hecho de la pérdida de poder de la organización va a ser uno de los aspectos importantes que van a marcar el resurgimiento de una producción académica más cercana a los conceptos de paz y reconciliación. 
Sin embargo, un factor clave será la política antiterrorista llevada a cabo por el expresidente Alberto Fujimori (Bowen, 2000).

Tras un periodo de alta controversia y mano dura, Fujimori logró cortar de raíz las acciones del grupo armado, que aparece como derrotado después de la detención de su líder, Óscar Ramírez, sucesor de Abimael Guzmán, detenido en 1992. Si bien Fujimori va a gozar de un gran apoyo dentro de la población peruana, sus acciones armadas y su estrategia para terminar con la presencia de Sendero Luminoso en gran parte del país generará una gran controversia. Su salida precipitada del país en el año 2000 traerá como consecuencia la creación de la Comisión de la Verdad y Reconciliación del Perú en el año 2001 que tendrá como objetivo el generar el relato y salvaguardar la memoria de los años mas duros de la violencia.

Como resultado de lo anterior, se generarán una serie de informes (Comisión de la Verdad y la Reconciliación, 2003a, 2003b, 2004) en los que se verán reflejados los conceptos de verdad apuntados anteriormente en este trabajo. Sin embargo, y aunque la comisión también tenga como objetivo la reconciliación, esta solamente se hará en un sentido de presentar el daño causado por las partes, siguiendo el enfoque histórico teatral presentado por Johan Galtung (1998). La existencia de esos informes y su divulgación impacta de manera decisiva en la producción intelectual referente al conflicto en el Perú, abriéndose a esta nueva faceta que ya no será evitada por los distintos autores. En efecto, un nuevo ciclo de trabajo académico surgirá, centrado en la importancia y el impacto de los procesos de memoria y restauración del trauma (Améry, 2004; Butler, 2010). Estos trabajos se unirán a una creciente corriente que buscará analizar de forma crítica el papel del Estado en el conflicto peruano.

Después de los informes de la Comisión de la Verdad y la Reconciliación, una serie de contribuciones arrojaron luz sobre los abusos realizados por las fuerzas del Estado (Hayner, 2001; Mora et al., 2001; Kruijt y Tello, 2003; Uceda, 2004). La realización de la posibilidad de que el Estado peruano pudiera haber cometido atrocidades en el marco del conflicto armado marcó la posibilidad de la aparición de estudios de este tipo, lo que llevaría a una reevaluación de los distintos trabajos realizados al respecto (Ríos y García de las Heras, 2019). La existencia de estos hechos, que hacían mas nublosa la diferencia entre grupo guerrillero y fuerzas del Estado es lo que genera la pregunta que domina este trabajo. Sin embargo, estas contribuciones no buscan generar una crítica al quehacer del Estado como un agente más del conflicto armado, sino presentar cómo el comportamiento particular de la fuerza pública en un momento concreto puede ser criticable. El objetivo de estos trabajos no es poner en un mismo nivel a los distintos actores del conflicto, lo que hubiera ayudado a la aparición de trabajos que genuinamente pudieran haber propuesto 
la idea de construcción de paz y reconciliación en el país, sino el proponer, desde un enfoque crítico, la acción particular de un gobierno concreto.

Los enfoques basados en la memoria y la descripción del relato serán los que marcarán el protagonismo de las contribuciones académicas a la explicación del conflicto peruano de manera más significativa. Estos análisis surgirán bajo el paraguas creado por los informes de la Comisión de la Verdad y la Reconciliación y buscarán poner sobre la mesa el traume y el sufrimiento causado por la violencia ligada al conflicto interno (De la Cadena, 2003; Degregori, 2003; Sandoval, 2003; Del Pino Huamán, 2008). Una característica de estos trabajos es la ausencia de trabajos puros que tengan que ver con la construcción de paz por la ausencia de académicos especialistas en ese campo.

La mayoría de las contribuciones al respecto son realizadas desde los campos de la antropología y desde la historia. Sin embargo, es útil resaltar que en los años posteriores a la publicación de los informes de la Comisión de la Verdad y la Reconciliación también han aparecido intentos de incluir los conceptos relacionados con la construcción de paz en la literatura referente al conflicto peruano. Dentro de esos trabajos cabe resaltar las contribuciones de Gustavo Gutiérrez y su propuesta de un modelo de reconciliación basado en las conclusiones del informe de la comisión (Gutiérrez, 2003) y la propuesta de Kimberly Theidon, quien analiza de forma crítica la política pública de reconciliación que existe en el Perú post-comisión, sugiriendo elementos muy cercanos al reconocimiento del otro como agente de construcción de paz (Theidon, 2004).

Finalmente, es importante mencionar los diversos estudios que han surgido analizando de forma crítica la historia del conflicto y su comportamiento, así como el papel de sus actores más importantes. Dentro de esta última categoría se encuentran los trabajos de Mario Fumerton y Simone Remijnse describiendo los distintos modelos de autodefensa civil que surgen en el Perú (Fumerton \& Remijnse, 2004) y el reciente trabajo de Jerónimo Ríos y Marté Sánchez exponiendo de forma sucinta y objetiva la historia de Sendero Luminoso (Ríos y Sánchez, 2018). El propio Jerónimo Ríos presenta un interesante estudio de reciente publicación, que explora los discursos y narrativas de antiguos combatientes de Sendero Luminoso sobre el actual proceso político peruano (Ríos, 2019). Este trabajo lanza un mensaje preocupante, al situar muy lejana la posibilidad de reconciliación en el país. La producción intelectual sobre el conflicto peruano también se ha visto enriquecida por trabajos de análisis riguroso sobre eventos acaecidos durante los años de la violencia (Cristóbal, 2003; Youngers, 2003; Ríos y García de las Heras, 2019), y que son trabajos que no hubieran podido surgir sin la contribución realizado por la Comisión de la Verdad y la Reconciliación. 
En resumen, desde el año 2001 han surgido una multiplicidad de trabajos que se han acercado mucho más a los conceptos de construcción de paz y reconciliación presentados anteriormente. Esto ha sido posible al cambio de política pública hacia la violencia ocurrido tras el gobierno de Alberto Fujimori y la creación de la Comisión de la Verdad y la Reconciliación. La mayoría de los trabajos han ido enfocados hacia los conceptos de memoria y tratamiento del trauma de los colectivos víctimas de la violencia, siguiendo los lineamientos de la mencionada comisión, aunque también ha surgido espacio para tratar, de forma crítica, el comportamiento de los actores estatales. De forma muy paulatina, además, han surgido trabajos que han buscado lanzar contribuciones hacia la construcción de paz en el país. Sin embargo, estos trabajos, actualmente $\mathrm{y}$ aun con todo, siguen siendo muy escasos.

\section{Conclusiones}

El caso peruano es una rara avis dentro de los estudios de caso de conflicto. Siendo un país con un largo periodo de violencia extrema, los estudios académicos han tocado poco el tema desde la perspectiva de los estudios de paz y conflicto. Este trabajo se hacía eco de las inquietudes presentadas por Carlos Iván Degregori en su obra Qué difícil es ser Dios sobre el motivo de tal falta de estudios. Sin embargo, la perspectiva presentada por el autor es bastante limitante. Si bien se plantea la necesidad de generar estudios referentes al conflicto peruano que ganen en calidad y profundidad analítica, las inquietudes presentadas por Degregori no reconocen el campo académico resultante los estudios de paz. Por ello se hace complicado presentar un marco de trabajo académico que utilice los conceptos de construcción de paz y reconciliación presentador por la literatura actual.

Este trabajo presenta dos partes claramente diferenciadas. Por un lado, se presentan los conceptos de construcción de paz y reconciliación tal y como se presentan dentro del cuerpo de estudios de paz. Para ello se explora el trabajo realizado por autores como Johan Galtung o John Paul Lederach, los grandes teóricos de la disciplina, así como las aportaciones más novedosas y contemporáneas al respecto. Si bien hay factores que diferencian cada enfoque, un aspecto que tienen en común las distintas definiciones de construcción de paz y reconciliación es la necesidad de analizar las causas base del conflicto, sus manifestaciones de violencia y presentar una serie de aspectos que avancen hacia una mejor gestión de esas causas.

Todas las definiciones de reconciliación incluyen la necesidad de presentar mecanismos que ayuden a una sociedad dividida a superar sus tensiones derivadas de la violencia para avanzar a un modelo de gestión del conflicto 
constructivo. Por ejemplo, Johan Galtung apunta a la necesidad de buscar medios que traten el trauma de las víctimas de la violencia, bien por medios de reconocimiento del relato y la memoria, medios que enfaticen el castigo a victimarios o el acercamiento entre las partes protagonistas del conflicto.

Normalmente, este tipo de estudios surgen en entornos de conflicto activo. En América Latina son característicos los estudios realizados durante la guerra civil en El Salvador, Guatemala u Honduras, pero, sin lugar a duda, el laboratorio de creación de trabajos relacionados con la paz y los conflictos es Colombia. Por lo tanto, es interesante comprobar como, en caso peruano, esto no se ha dado. En la segunda parte del trabajo se presenta como, durante los años de la violencia (1980-1999) los trabajos académicos que tratan el conflicto interno en el Perú se centran en describir la violencia y presentar la estrategia y existencia de Sendero Luminoso como una anomalía que debe ser reparada. En efecto, los estudios se centrarán en discutir la legitimidad de la organización armada, la necesidad de sus métodos y el daño causado por su presencia en el territorio. Degregori lanza unas ideas al respecto, como la naturaleza poscolonial del pensamiento intelectual peruano o la distancia que existe entre lo rural y lo urbano en el país.

Este enfoque cambia durante el periodo que sigue al descabezamiento final de Sendero Luminoso y a la caída del gobierno de Alberto Fujimori. Desde el año 2000 en adelante aparecerán nuevas formas de entender la violencia en el Perú donde se pueden apreciar algunos enfoques teóricos centrados en la construcción de paz y la reconciliación. Estos estudios surgirán bajo el paraguas de los informes realizados por la Comisión de la Verdad y la Reconciliación creada por el presidente provisional del país, Valentín Paniagua en junio de 2001.

La existencia de la Comisión influirá decisivamente en la naturaleza de la producción académica relacionada con la violencia en el Perú, generando la aparición de muchas contribuciones centradas en el papel de la memoria y el relato. Además, aspectos que antes no se habían abordado, dejarán de estar vedados. Es importante mencionar en este apartado los estudios críticos que surgen de la labor y accionar del Estado durante los años de la violencia, las propuestas de paz mucho más cercanas a los conceptos de construcción de paz y reconciliación defendidos por los teóricos de los estudios de paz y los análisis profundos y críticos de los hechos históricos que acaecieron en las décadas de los 80 y 90 . Dentro de este apartado merece resaltar los trabajos que analizan el nacimiento e historia de Sendero Luminoso, aportando una rigurosidad que no se encontraba anteriormente.

Este trabajo no busca explicar las razones por las que existe un vacío intelectual sobre los estudios de paz referentes al caso peruano, sobre todo durante los años más duros de la violencia. Lo que se busca es presentar este 
hecho y lanzar unas preguntas al respecto. Se aprecia desde el año 2001 un aumento en las contribuciones sobre paz en el Perú, pero aún se observa una escasez en la producción académica al respecto. Este trabajo espera poder sentar las bases para avanzar en dos aspectos diferenciados: La visión crítica del por qué no se ha realizado el ejercicio de contribución intelectual al conflicto peruano basada en los estudios de paz y la generación de trabajos académicos al respecto. El expandir el campo de los estudios de paz en el Perú puede ayudar a generar escenarios que ayuden a la reconciliación nacional de un país que aun siente, de manera muy reciente, los efectos de una violencia devastadora.

\section{Referencias bibliográficas:}

Ambos, K. (1999). Impunidad, Derchos Humanos y Derecho Penal Internacional. Nueva Sociedad, 161, 86-102.

Améry, J. (2004). Más allá de la culpa y la expiación: Tentativas de superación de una víctima de la violencia. Madrid: Pre-Textos.

Ansión, J. (1982). ¿Es luminoso el camino de Sendero? El Caballo Rojo, 108, 4-5.

Ansión, J. (1989). Pishtacos: De verdugos a sacaojos. Lima: Tarea.

Ansión, J. (1992). La escuela en tiempos de guerra. Lima: CEAPAZ.

Apter, D. (1993). Democracy, Violence and Emancipatory Movements: Notes for a Theory of Inversionary Discourse. Nueva York: UNRISD.

Arce Borja, L. (Ed.). (1989). Guerra popular en el Perú: El pensamiento de Gonzalo. Lima: L.A. Borja.

Ashplant, T. G., Dawson, G., \& Roper, M. (Eds.). (2004). Commemorating War. The Politics of Memory. Londres: Routledge.

Ball, N. (2001). The challenge of rebuilding war-torn societies. En C. Crocker, F. Osler Hampson, \& P. All (Eds.), Turbulent Peace. The challenges of managing international conflict. Washington: USIP.

Basombrio, C. (1994). Para la historia de una guerra con nombre: ¡Ayacucho! Ideele, 62, 27-33.

Berg, R. (1986). Sendero Luminoso and the peasantry of Anahuaylas. Journal of Interamerican Studies and World Affairs, 28(4), 165-196.

Biondi, J., \& Zapata, E. (1989). El discurso de Sendero Luminoso: Contratextos educativos. Lima: CONCYTEC.

Bolaños, A. (1992). Proyecto educación y cultura de paz. Educación, Volumen Extraordinario, 211-218.

Bowen, S. (2000). El expediente Fujimori: El Perú y su presidente 1990-2000. Lima: Perú Monitor. 
Burga, M. (1988). Nacimiento de una utopía: Muerte y resurrección de los incas. Lima: Instituto de Apoyo Agrario.

Butler, J. (2010). Marcos de guerra: Las vidas lloradas. Barcelona: Paidós.

Castañeda, J. (1993). La utopía desarmada: Intrigas y dilemas de la izquierda en América Latina. México: Joaquín Mortiz.

Comisión de la Verdad y la Reconciliación. (2003a). Informe final de la Comisión de la Verdad y Reconciliación en el Perú. CVR.

Comisión de la Verdad y la Reconciliación. (2003b). Para que nunca se repita. Informe final de la CVR. APRODEH.

Comisión de la Verdad y la Reconciliación. (2004). Hatun Willakuy. Versión abreviada del informe final de la Comisión de la Verdad y Reconciliación. CVR.

Coser, L. (1956). The functions of social conflict. Londres: Routledge.

Cristóbal, J. (2003). Uchuraccay o el rostro de la barbarie. Lima: Editorial San Marcos.

Crocker, C., Osler Hampson, F., \& All, P. (2001). Turbulent Peace. The challenge of managing international conflict. Washington: USIP.

Crocker, C., Osler Hampson, F., \& All, P. (2015). Managing Conflict in a World Adrift. Washington: USIP.

Dahrendorf, R. (1968). Hacia una teoría del conflicto social. En E. Etzioni \& A. Etzioni (Eds.). Los cambios sociales. México: Fondo de Cultura Económica.

De la Cadena, M. (2003). Escribir otra historia del Perú. Cuestión de Estado, $32,12-13$.

De Soto, H. (1986). El otro sendero: La revolución informal. Lima: Instituto de Libertad y Democracia.

Degregori, C. I. (1996). Las rondas campesinas y la derrota de Sendero Luminoso. Lima: IEP.

Degregori, C. I. (1997). Perú. Más allá de la toma de rehenes. Nueva Sociedad, $148,6-11$.

Degregori, C. I. (2003). Jamás tan cerca arremetió lo lejos: Memoria y violencia política en el Perú. Lima: IEP.

Degregori, C. I. (2011). Qué difícil es ser Dios. EL partido comunista del PerúSendero Luminoso y el conflicto armado interno en el Perú: 1980-1999. Lima: IEP.

Del Pino, P. (2008). Looking to the Government: Community, Politics and the Production of Memory and Silences in Twentieth-Century Peru, Ayacucho [PhD Dissertation]. University of Wisconsin-Madison.

DESCO. (1989). Violencia política en Perú, 1980-1988. Lima: DESCO.

Díaz Bazo, C. (1996). Una estrategia de formación de maestros para la paz. Educación, 5(10), 207-215. 
Dreyfus, P. G. (1999). When all the evils come together: Cocaine, corruption, and Shining Path in Peru's Upper Huallaga Valley, 1980-1995. Journal of Contemporary Criminal Justice, 15(4), 370-396.

Fisas, V. (2002). Cultura de paz y gestión de conflictos. Barcelona: Icaria.

Fisas, V. (2010). ;Alto el fuego! Manual de procesos de paz. Barcelona: Icaria. Fitzduff, M. (2002). Beyond Violence: Conflict Resolution Processes in Northern Ireland. Nueva York: UN University Press.

Fumerton, M., \& Remijnse, S. (2004). Civil Defense Froces: Peru's Comités de Autodefensa Civil and Guatemala's Patrullas de Autodefensa Civil in Comparative Perspective. Koonings, K. \& Kruijt, D. (Eds.). Armed Actors: Organised Violence and State Failure in Latin America. Londres: Zed Books.

Gago, E. (2016). Una aproximación teórica a los conceptos de construcción de paz y posconflicto armado. En Barreto, M. (Ed.), Experiencias internacionales de paz lecciones aprendidas para Colombia. Bogotá: Universidad Jorge Tadeo Lozano.

Galtung, J. (1998). After violence: 3R, Reconstruction, Reconciliation, Resolution. Coping with visible and invisible effects of war and violence. Oslo: Transcend.

Galtung, J. (2003). Paz por medios pacíficos. Paz y conflicto, desarrollo y civilización. Gernika Gogoratuz.

Garcia-Duran, M. (Ed.). (2004). Alternativas a la guerra. Iniciatives y procesos de paz en Colombia. Bogotá: CINEP.

Giesecke, M. (1993). Cultura de paz y enseñanza de historia. En EcuadorPerú. Horizontes de la negociación y el conflicto. Quito/Lima: FLACSO/ DESCO.

González Marrero, S., \& Harto de Vera, F. (1994). Transición y elecciones en El Salvador. America Latina Hoy, 8, 73-80.

Hayner, P. (2001). Unspeakable Truth: Confronting State Terror and Atrocities. Londres: Routledge.

Kaldor, M. (1998). New \& Old Wars. Londres: Polity Press.

Kaufman, S. (1998). Sobre violencia social, trauma y memoria. Social Science Research Council. Memoria Colectiva y Represión: Perspectivas comparativas sobre el proceso de democratización en el Cono Sur de América Latina. Montevideo, Uruguay.

Kruijt, D. \& Tello, M. del P. (2003). De los reformistas militares a la dictadura civil: la política militar peruana desde los años sesenta hasta el presente. En. Koonings, K y Kruijt, D. (Eds.), Ejércitos politicos: Las Fuerzas Armadas y la construcción de la nación en la era de la democracia. Lima: IEP.

Lederach, J. P. (1998). Construyendo la paz. Reconciliación sostenible en sociedades divididas. Gernika Gogoratuz. 
Lederach, J. P. (2005). The Moral imagination: The art and soul of building peace. Oxford: Oxford University Press.

Lederach, J. P., \& Lederach, A. J. (2014). Cuando la sangre y los huesos claman. Travesías por el paisaje sonoro de la curación y la reconciliación. Gernika Gogoratuz.

Mac Ginty, R. (2006). No War, No Peace: The rejuvenation of stalled Peace Processes and Peace Accords. Londres: Palgrave.

Mac Gregor, F. (1986). Cultura de Paz. París: UNESCO.

Mac Gregor, F., Rouillón, J. L., \& Rubio, M. (Eds.). (1985). Siete ensayos sobre la violencia en el Perú. Lima: Fundación Friedrich Ebert, Asociación Peruana de Estudios e Investigaciones para la Paz.

Mason, T. D., \& Campany, C. (1995). Guerrillas, Drugs and Peasants: The Rational Peasant and the War on Drugs in Peru. Terrorism and Political Violence, 7(4), 140-170.

Medina Gallego, C. (2010). FARC-EP y ELN. Una historia politica comparada (1958-2006). Bogotá. Universidad Nacional de Colombia.

Mora, D., Rospigliosi, F., Abad Yupanqui, S. y Basombrio, C. (2001). Las fuerzas armadas en la transición democrática en el Perú. Lima: IEP.

Nordquist, K.-A. (2018). La reconciliación como política. El concepto y su práctica. Bogotá: Universidad Javeriana.

Özerdem, A., \& Lee, S. Y. (2016). Peacebuilding: An Introduction. Londres: Routledge.

Özerdem, A., \& Mac Ginty, R. (2019). Comparing Peace Processes. Londres: Routledge.

Palmer, D. (1992). Shining Path of Peru. Nueva York: St Martin's Press.

Poole, D. y Rénique, G. (1991). The new chroniclers of Peru: U.S. Scholars end their «Shining Path» of Peasant rebellion. Bulletin of Latin American Research, 10(1), 133-191.

Ramsbothan, O., Woodhouse, T., \& Miall, H. (2015). The Contemporary Conflict Resolution Reader. Londres: Polity Press.

Richmond, O. (2005). The transformation of peace. Londres: Palgrav.

Ríos, J. (2019). Narratives about Political Violence and Reconciliation in Peru. Latin American Perspectives, 46(5), 44-58.

Ríos, J. y Gago, E. (2015). Entre los debates teóricos del posconflicto y la realidad colombiana. Una aproximación al caso de Nariño. En Niño, C. (Ed.), Perspectivas y prospectivas de la seguridad en Colombia. Bogotá: Universidad Santo Tomás.

Ríos, J. y Gago, E. (2018). La paz territorial en Colombia: Un desafío para la institucionalidad local. Papers. Revista de Sociología, 103(2), 281-302.

Ríos, J. y García de las Heras, M. (2019). "Experiencias sobre el terrorismo de estado en Perú”. Revista Universitaria de Historia Militar, 8(17), 71-96. 
Ríos, J. y Sánchez, M. (2018). Breve historia de Sendero Luminoso. Madrid: Libros de la Catarata.

Sandoval, P. (2003). El olvido está lleno de memoria: Juventud universitaria y violencia política en el Perú, La matanza de estudiantes de La Cantuta. En Degregori, C.I. (Ed.). Jamás tan cerca arremetió lo lejos: Memoria y violencia politica en el Perú. Lima: IEP.

Stromseth, J. E. (2015). Peacebuilding and transitional justice. En Crocker, C., Osler Hampson, F. y All, P. (Eds.). Managing conflict in a world adrift. Washington: USIP.

Strong, S. (1992). Shining Path: Terror and Revolution in Peru. Nueva York: Times Books.

Theidon, K. (2004). Entre prójimos: El conflicto armado interno y la política de reconciliación en el Perú. Lima: IEP.

Uceda, R. (2004). Muerte en el Pentagonito: Los cementerios secretos del ejército peruano. Lima: Planeta.

Urbano, H. (Ed.). (1991). Poder y Violencia en los Andes. Cusco: Centro de Estudios Regionales Andinos Bartolomé de Las Casas.

Valenzuela, P. (2014). Consideraciones sobre un proceso de paz sobre el ELN. En De Currea Lugo, V. (Ed.) ¿Por qué negociar con el ELN? Bogotá: Universidad Javeriana.

Vinyamata, E. (2001). Conflictología. Teoría y práctica de la resolución de conflictos. Barcelona: Ariel.

Woodhouse, T., Ramsbothan, O. y Miall, H. (2011). Contemporary conflict resolution. Londres: Polity Press.

Youngers, C. (2003). VIolencia política y sociedad civil en el Perú: Historia de la Coordinadora Nacional de Derechos Humanos. Lima: IEP. 